\title{
A Fuzzy Measurement Model of Mobile Internet Security Hong Chen ${ }^{a}$, Chen Wang ${ }^{b}$,Zhengying Cai ${ }^{\text {** }}$
}

College of Computer and Information technology, China Three Gorges University, Yichang 443002, China

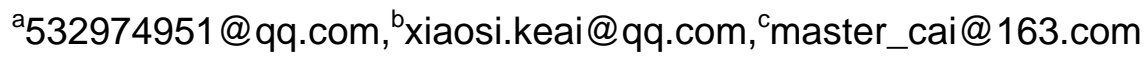

*Corresponding author

Keywords: Security measurement, Fuzzy Entropy, risk assessment, Mobile Internet.

\begin{abstract}
This article discusses the security assessment methodology of mobile internet. Above all, the impact of risk environment on network security is analyzed. In accordance with the actual situation of mobile internet, each risk factor is identified and measured by 3 dimensional indexes including its probability, loss and risk impact. The next, based on them, a fuzzy entropy measurement method for mobile internet security assessment is proposed. Through the fuzzy logic and risk entropy weight, the security of mobile internet can be calculated to evaluate the entire system. At last, an example verified the convenience of proposed method for the security assessment of mobile internet.
\end{abstract}

\section{Introduction}

With the rapid development of mobile internet, the issue on network security or risk assessment becomes very important[1]. The character of the mobile internet is to transmit information by air as the transmission medium, so that the listener may be a non-contact. Therefore the safety problem of mobile internet is different from that of wired network, and becomes particularly prominent [2]. Because risk assessment of mobile internet security is an important part to the wireless network application [3], several researches discussed the security assessment method of mobile internet [4],[5].

Because of the risk factors, it is difficult to quantify uncertainties[6]. Here a risk assessment method is proposed by fuzzy logic method, providing us a qualitative evaluation tool to determine which risk factors need to take measures or to be controlled. Based on the actual situation of the mobile internet security, the fuzzy logic method is improved by entropy which can be calculated by fuzzy reference of the entire network system.

\section{Fuzzy measurement of mobile internet security}

Fuzzy measurement is used to assess the risk of mobile internet system, as shown in Fig.1.

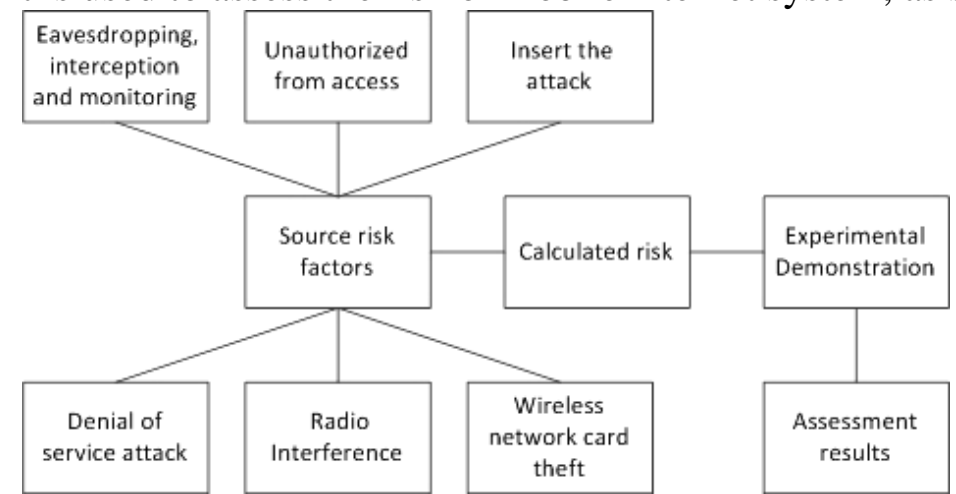

Fig. 1 Fuzzy security assessment

The security assessment system is not only a function of the probability $P$ of risk events, but also a function of risk impact $C$ arising from the event, and a function of uncontrollable loss $U$ of risk events. By measuring probability $P$, impact $C$ and uncontrollability $U$, the subscript $f$ denotes the risk event $s$ not occurred. Then there is the following relationship: $P_{f}=1-P_{s}, C_{f}=1-C_{s}$, 
$U_{f}=1-u_{s}$.

Risk $R$ is an actually occurred risk event, the consequences of risk events and uncontrollability can be estimated.

$R=f(s)=1-p_{f} c_{f} u_{f}=1-\left(1-p_{s}\right)\left(1-c_{s}\right)\left(1-u_{s}\right)=p_{s}+c_{s}+u_{s}-p_{s} c_{s}-p u_{s}-c u-p_{s} c_{s} u_{s}(1)$

According to risk equation (1), only the $P_{s}, C_{s}$ and $U_{s}$ are needed to calculate the system risks. Because of the uncontrollability and ambiguity of the risk events, the risk impact and the consequences of event occurred, the fuzzy logic method is employed to calculate $P_{s}, C_{s}$ and $U_{s}$.

It is assumed that the fuzzy evaluation set is $U=\left\{u_{1}, u_{2}, \ldots, u_{n}\right\}$. Then the expert reference of risk assessment can be constructed according to the risk factors by different criteria in hierarchy layer. For each risk factors, its evaluation level is certain, and the evaluation set of established class set is defined as $V=\left\{v_{1}, v_{2}, \ldots, v_{m}\right\}$, which is also called as the risk factor evaluation sets. Based on them, a second layer of different criteria of risk factors can be evaluated.

The expert judgment set $V$ refers to the evaluation of various factors in the factor set $U$, giving us the various factors reviews, and constructs a fuzzy mapping $f: u \rightarrow F(V), F(V)$ which is all fuzzy sets of $V, U_{i} \rightarrow f\left(u_{i}\right)=\left(r_{i 1}, r_{i 2}, \ldots, r_{i m}\right) \in F(V)$, where the map $f$ represent risk factors for judging the degree of centralized support for each comment $U_{i}$. For the judger, risk factors to make $V$ set membership vector of $U_{i}$ is represented as $R_{i}=\left\{r_{i 1}, r_{i 2}, \ldots, r_{i m}\right\}, i=1,2, \ldots, n$.

With respect to the probability, impact and uncontrollability, different membership matrix of risk factors can be gotten as $R_{p}, R_{c}$ and $R_{u}$ respectively.

Calculating the probability of the risk event, corresponding weight vector of each factors are $A_{p}=\left(a_{1}, a_{2}, \ldots, a_{n}\right)$. As the evaluation set $V$, each indexed give appropriate weight for index weight vector $B_{p}=\left(b_{1}, b_{2}, \ldots, b_{m}\right)$. Then the probability of the risk event is $P_{s}=A_{p} R_{p} B_{P}^{T}$. Now the impact of risk events is generated by various factors, and the corresponding weight vector is $A_{i}=\left(a_{1}, a_{2}, \ldots, a_{n}\right)$. Similarly, each index gives appropriate weight for the index weight vector $B_{i}=\left(b_{1}, b_{2}, \ldots, b_{m}\right)$. The impact of risk events is generated as $C_{s}=A_{i} R_{i} B_{i}$. In calculating the uncontrollability of risk events, corresponding weight vector of each factor is $A_{u}=\left(a_{1}, a_{2}, \ldots, a_{n}\right)$. At the same time each index gives appropriate weight for the index weight vector $B_{u}=\left(b_{1}, b_{2}, \ldots, b_{m}\right)$. Therefore the uncontrollability of risk events is $U_{s}=A_{u} R_{u} B_{u}^{T}$.

Based on the calculated $P_{S}, C_{s}$ and $U_{s}$ above, the system risk of $R$ can be calculated. Generally it is believed that $\mathrm{R}>0.7$ for high-risk systems, and $\mathrm{R}<0.3$ for low-risk systems, suitable for general mobile internet systems.

\section{Entropy weight of risk factor}

Based on the membership matrix of risk factors from expert judgment, entropy can be set by extreme resistance. Often $P_{i}$ is closer to the entropy, if it is greater, then the risk assessment for systemic risk factors is greater in uncertainty. Thus, according to centralized evaluation of various risk factors, information entropy is used to support each index $r_{i j}$ by the calculated index weights.

The relative entropy importance of risk factor $U_{i}$ is measured by the following equation $H=-\sum_{i=1}^{n} p_{i} L_{i} P_{i}$, where $r_{i j}(j=1,2, \ldots, m)$ is closer to the entropy. If it is greater, then the systemic risk assessment of risk factors $U_{i}$ is greater in uncertainty. When values $r_{i j}(j=1,2, \ldots, m)$ equals 
to the maximum entropy of $H_{\max }=L_{n} m$, and $H_{\max }$ is normalized, we will get a measure of entropy $e_{i}=-\frac{1}{L_{n} m} \sum_{j=1}^{m} r_{i j} L_{n} r_{i j}$ of the relative importance of risk factors $U_{i}$.

When $r_{i j}(j=1,2, \ldots, m)$ is equal to the value of a maximum entropy $e_{i}$, the value of $e_{i}$ satisfies $0 \leqslant e_{i} \leqslant 1$. Since the maximum entropy represents the contribution of risk factors on the risk assessment of the minimum system, the weight to determine risk factors for $U_{i}$ can be measured as $1-e_{i}$. It can be further normalized to obtain the weighted value of the fuzzy risk factors $U_{i}$ $\cong_{i}=\frac{1}{n-E}\left(1-e_{i}\right)$, where $E=\sum_{i=1}^{n} e_{i} \cong_{i}$ satisfies $0 \leq \cong_{i} \leq 1, \sum_{i=1}^{n} \cong_{i}=1$.

\section{Experiment}

To verify the proposed method, a simulated experiment is employed, and system configuration includes computer processor Intel (R) Core (TM) 2 Quad CPU Q9500 @ 2.83GHz 2.83 GHz, and installed memory 4.00GB (3.00GB available). The system environment is 32-bit operating Windows 7 Ultimate and MATLAB 7.0. Figure 3 shows the experimental results.

As we can see from Fig.1, during the rising stage, in the traditional measurement method, it is found to violently disturbed with obvious error. However the fuzzy method can increase rapidly in the initial stage, and then keeps at relatively stable 0.4, lastly it rapidly rises again after a period of time of 0.2, maintains in higher level of 0.62. Apparently the fuzzy measurement method is more suitable for the human expression habits for risk measurement.

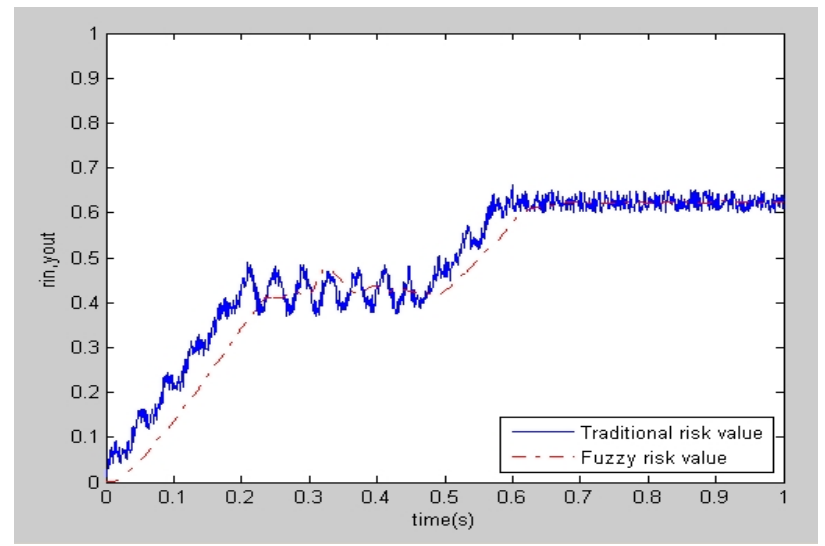

Fig. 2 Fuzzy security measurement

\section{Conclusions}

In this paper, a security assessment method of the mobile network is built here using fuzzy mathematics, which is in accordance with the actual situation of its mobile network. Risk factors are calculated by risk probability, impact and uncertainties, and weight vector of risk factors are used to overcome the direct assignment of subjectivity by given information entropy.

\section{Acknowledgements}

This research was supported by the National Natural Science Foundation of China (No. 71471102), and Key Projects of Science and Technology Research Program of Hubei Provincial Department of Education in China (Grant No. D20101203). 


\section{References}

[1] Keith, Mark J .; Thompson, Samuel C .; Hale, Joanne Nformation disclosure on mobile devices:. Re-examining privacy calculus with actual user behavior $[\mathrm{J}]$.International journal of human-computer studies, 2013,71 (12): 1163-1173.

[2] Tang Hong-Bo; Yang Zhi-Xing; Chen Long Analysis on Security Issues for Mobile P2P Networks [C] 8th International Conference on Wirelesommunications, Networking and Mobile Computing (WiCOM), 2012:21-23.

[3] Wang Qin; Zhi Fenhe; Ao Jinghai Design and Security Analysis of Mobile Identity Authentication Protocol [C] 7th International Conference on MEMS, NANO and Smart Systems (ICMENS 2011), 2011:04-06.

[4] Wang, Nan; Shen, Xiao-Liang; Sun, Yongqiang.Transition of electronic word-of-mouth services from web to mobile context: A trust transfer perspective [J] .Decision support systems, 2013,54 (3) : 1394-1403.

[5] Piao, Chunhui; Dong, Suqin; Cui, Liang A Novel Scheme on Service Recommendation for Mobile Users Based on Location Privacy Protection [C] .International Conference on e-Business Engineering (ICEBE 2013), 2013:11-13.

[6] El-degwy, Hossam; Kelash, Hamdy M.; Faragallah, Osama S. Building a Secure E-Transaction System Based on Mobile Agent [C] 8th International Computer Engineering Conference (ICENCO)

- Today Information Society What's next, 2012 : 29-30. 